\title{
छs \\ Generalized algorithm for control of numerical dispersion in explicit time-domain electromagnetic simulations
}

\author{
Benjamin M. Cowan, ${ }^{*}$ David L. Bruhwiler, ${ }^{\dagger}$ John R. Cary, and Estelle Cormier-Michel \\ Tech-X Corporation, Boulder, Colorado 80303, USA \\ Cameron G. R. Geddes \\ LOASIS program, Lawrence Berkeley National Laboratory, Berkeley, California 94720, USA
}

(Received 26 July 2012; published 4 April 2013)

\begin{abstract}
We describe a modification to the finite-difference time-domain algorithm for electromagnetics on a Cartesian grid which eliminates numerical dispersion error in vacuum for waves propagating along a grid axis. We provide details of the algorithm, which generalizes previous work by allowing 3D operation with a wide choice of aspect ratio, and give conditions to eliminate dispersive errors along one or more of the coordinate axes. We discuss the algorithm in the context of laser-plasma acceleration simulation, showing significant reduction — up to a factor of 280, at a plasma density of $10^{23} \mathrm{~m}^{-3}$-of the dispersion error of a linear laser pulse in a plasma channel. We then compare the new algorithm with the standard electromagnetic update for laser-plasma accelerator stage simulations, demonstrating that by controlling numerical dispersion, the new algorithm allows more accurate simulation than is otherwise obtained. We also show that the algorithm can be used to overcome the critical but difficult challenge of consistent initialization of a relativistic particle beam and its fields in an accelerator simulation.
\end{abstract}

DOI: 10.1103/PhysRevSTAB.16.041303

PACS numbers: 41.75.Jv, 41.60.- $-\mathrm{m}$

\section{INTRODUCTION}

The finite-difference time-domain (FDTD) algorithm $[1,2]$ is a well-established technique for updating electromagnetic fields on a Cartesian grid. The algorithm can easily be shown to be stable as long as the time step does not exceed the Courant limit (discussed below). It is explicit, and is therefore computationally efficient and straightforward to implement and parallelize. For these reasons, FDTD has become very widely used, with applications in rf devices, plasma physics, and optics as well as particle accelerators. However, it is well known that FDTD exhibits numerical dispersion error for waves that are not propagating along a grid diagonal. In particular, for FDTD simulations in more than one dimension, the Courant condition requires that the time step $\Delta t$ satisfy $c \Delta t<\Delta x$, where $\Delta x$ is the grid spacing in any direction and $c$ is the speed of light in vacuum. As a result, waves propagating along a grid axis have numerical group velocity $<c$.

Numerical dispersion errors have serious consequences in models where physical phenomena propagate quickly along an axis, as is often the case in particle accelerator simulations. One consequence of this is that a relativistic particle beam emits numerical Čerenkov radiation due to its

\footnotetext{
*benc@txcorp.com

${ }^{\dagger}$ Present address: 1348 Redwood Avenue, Boulder, CO 80304.
}

Published by the American Physical Society under the terms of the Creative Commons Attribution 3.0 License. Further distribution of this work must maintain attribution to the author(s) and the published article's title, journal citation, and DOI. velocity being larger than the numerical speed of light on the grid. In addition, the self-fields of such beams are incorrect if the numerical dispersion is not controlled. These consequences adversely affect simulations of beamstructure interactions, including wakefield calculations [3].

Another accelerator application affected by numerical dispersion, which we will consider in detail here, is that of laser-plasma acceleration (LPA) [4-6]. In LPA, a relativistically intense laser pulse propagates through an underdense plasma, the ponderomotive force of the laser fields driving an electrostatic plasma wave. The resulting plasma wakefields can be orders of magnitude stronger than those available in conventional accelerators. The plasma wave has a wavelength characterized by the plasma wavelength $\lambda_{p}=\sqrt{\pi / n r_{e}}$, where $n$ is the electron number density and $r_{e}=e^{2} / 4 \pi \epsilon_{0} m c^{2}$ is the classical electron radius; here $\epsilon_{0}$ is the permittivity of free space and $-e$ and $m$ are the electron charge and mass, respectively. The intensity of the laser pulse is characterized by the normalized vector potential $a_{0}=e A_{0} / m c$, where $A_{0}$ is the peak value of the (unnormalized) vector potential of the laser field; a relativistic laser field is defined by $a_{0} \gtrsim 1$, which implies that an electron can be accelerated to relativistic velocities in a single optical cycle. In the linear regime, where $a_{0} \ll 1$, and in the 1D limit, the laser pulse has a group velocity approximately $v_{g}=c \sqrt{1-\lambda_{p}^{2} / \lambda_{0}^{2}}$, where $\lambda_{0}$ is the laser wavelength. This general scaling for the group velocity also holds as an approximation for the nonlinear regime $[7,8]$. A highly relativistic electron bunch moving at $\sim c$ will therefore advance with respect to the plasma wave phase moving at $v_{g}<c$, and after a sufficiently long 
propagation distance will leave the accelerating phase of the wake. This process is called dephasing and is one limitation on the length of an LPA stage. Dephasing plays a key role in determining the optimal stage length in highenergy LPA collider designs [9] and is sensitively dependent on the laser pulse group velocity, and hence dispersion. Significant theoretical and computational work has gone into LPA stage simulations, and has led to the development of new algorithms [10-12].

Laser-plasma acceleration has gained rapidly increasing interest since several years ago, when high-quality electron beams were produced by self-trapping and accelerated to $\sim 100 \mathrm{MeV}$ in a few millimeters [13-15]. Since then, efforts have focused on obtaining beams useful for highenergy particle colliders [9] and radiation sources [16-19]. This involves developing longer acceleration stages to achieve greater total energy gain, and controlling the injection process for higher beam quality. Recently, acceleration to $1 \mathrm{GeV}$ in a few centimeters was demonstrated [20]. In addition, experiments have shown that the quality of the electron beam can be improved by tailoring the plasma density profile in the injection process separately from the accelerating structure $[21,22]$ or by using colliding laser pulses [23].

Simulations have played a significant role in the development of LPA. In particular, the particle-in-cell (PIC) method, a well-established algorithm for self-consistently modeling charged particles in electromagnetic fields [24,25], has been critical to the understanding of the LPA processes. The PIC algorithm uses FDTD to update the electromagnetic fields, while tracking particles in continuous phase space. PIC simulations were used to predict injection of quasimonoenergetic bunches in self-trapping LPA experiments [26], and continue to be used extensively to understand the complex dynamics of the injection process [27-30].

Future numerical explorations of LPA present difficulties for PIC simulations, due in part to the numerical dispersion inherent in the FDTD algorithm. Acceleration to higher energies in a single plasma stage uses lower plasma densities, and therefore longer plasma wavelengths, to achieve group velocities closer to $c$ and delay dephasing effects. In that regime, numerical dispersion can be significant compared to physical dispersion due to the plasma [27], and lead to dephasing errors. Simulating longer, lower-density LPA stages also dramatically increases the computational resources required, due to the separation of length scales involved: An explicit FDTD simulation must encompass a longitudinal length $\sim \lambda_{p}$ and propagate over the order of the dephasing length $L_{d} \sim \lambda_{p}^{3} / \lambda_{0}^{2}$, while at the same time resolving the laser wavelength. In addition, the selftrapping process is a threshold phenomenon that is highly sensitive to physical parameters, in particular the phase velocity of the wake, which is equal to $v_{g}$. Therefore numerical errors such as dispersion effects can significantly alter the results of a simulation. Since the longitudinal direction has the smallest grid spacing, in order to resolve the laser wavelength $\lambda_{0} \ll w_{0}$, where $w_{0}$ is the spot size, and thus dominates the Courant limit of the time step, increasing the grid spacing can reduce the computational time quadratically. However, doing so increases the dispersion error in the Yee algorithm. Correct dispersion is also vital to the boostedframe technique [31], which can enable simulations of long, low-density stages with manageable computational cost.

Because of the deleterious effects of numerical dispersion in FDTD, efforts have been made to develop modified dispersion algorithms that exhibit no numerical dispersion for waves propagating along a grid axis. One method that has been employed artificially adjusts material parameters to compensate for dispersion [32]. For accelerator applications, several modifications to FDTD have been described that correct for numerical dispersion using implicit methods [3,33]. Because LPA simulations tend to be quite large scale (using thousands of processor cores), an explicit algorithm is desired for greater computational efficiency. Such an algorithm has been described in 2D [34] and in 3D for cubic cells [35]. These algorithms have been explored for LPA as a means of reducing noise in boosted-frame simulations [31]. Since lab-frame LPA simulations typically use noncubic, high aspect ratio cells due to the fact that the laser wavelength need only be resolved in the longitudinal direction, a general modified dispersion algorithm that can work with noncubic cells in 3D is desirable.

In the following section, we describe a generalizationthat we call controlled dispersion-of the algorithm in $[34,35]$ that allows a semi-infinite range of cell aspect ratio, and discuss its numerical dispersion properties. In Sec. III we demonstrate mitigation of group velocity errors in the linear regime with a laser pulse in a plasma channel; in Sec. IV we show the benefits of controlled dispersion simulations of realistic high-energy LPA stages. Our tests involve 3D simulations, to which [34] does not apply, and use a nonunity cell aspect ratio, to which [35] does not extend; thus, we compare the controlled dispersion against standard FDTD. Then, in Sec. V, we show how this controlled dispersion algorithm allows consistent initialization of a particle bunch in an accelerator simulation.

\section{ALGORITHM AND DISPERSION RELATION}

\section{A. Description of the algorithm}

Following [34,35], we modify the FDTD algorithm for controlled dispersion by smoothing the fields in one of the Maxwell curl updates in the directions transverse to the finite-difference direction. In our implementation we choose to smooth the electric fields for the magnetic field update; as we will see, the same dispersion properties would result from the opposite choice. Our update equations are then

$$
D_{t} \mathbf{B}=-\nabla^{\prime} \times \mathbf{E}, \quad D_{t} \mathbf{E}=c^{2} \boldsymbol{\nabla} \times \mathbf{B}-\frac{\mathbf{J}}{\epsilon_{0}},
$$


where $\mathbf{J}$ is the electric current deposited from particle motion. Here $D_{t}$ is the finite-difference time derivative, $\nabla \times$ is the standard finite-difference curl operator, and $\nabla^{\prime} \times$ is the modified curl operator. Because our modification to the curl operator involves applying smoothing transverse to the finite-difference direction, we have for instance that when computing $\partial E_{y} / \partial x, E_{y}$ is smoothed in the $y$ and $z$ directions. This is equivalent to applying a smoothing operator before the numerical derivative operator. The electric field is only smoothed for the update of the magnetic field; the smoothed fields are not stored for the next time step.

Suppose $D_{i}$ is the numerical derivative operator in the $x_{i}$ direction for each $i$. Then for the modified curl we use $D_{i} S_{i}$ in place of $D_{i}$, where $S_{i}$ is the smoothing operator for the $x_{i}$ derivative. We now define the smoothing operator $S_{x}$; in the following development similar relations hold for cyclic permutations of the coordinate indices. For an arbitrary $\mathbf{E}$ field component $\psi$, with $\left.\psi\right|_{i, j, k}$ its value in cell $(i, j, k)$, we let

$$
\begin{aligned}
\left.\left(S_{x} \psi\right)\right|_{i, j, k}= & \left.\alpha_{x} \psi\right|_{i, j, k}+\beta_{y}\left(\left.\psi\right|_{i, j+1, k}+\left.\psi\right|_{i, j-1, k}\right) \\
& +\beta_{z}\left(\left.\psi\right|_{i, j, k+1}+\left.\psi\right|_{i, j, k-1}\right) \\
& +\gamma_{y z}\left(\left.\psi\right|_{i, j+1, k+1}+\left.\psi\right|_{i, j+1, k-1}\right. \\
& \left.+\left.\psi\right|_{i, j-1, k+1}+\left.\psi\right|_{i, j-1, k-1}\right) .
\end{aligned}
$$

Here the $\alpha, \beta$, and $\gamma$ are coefficients satisfying $\alpha_{x}+2 \beta_{y}+2 \beta_{z}+4 \gamma_{y z}=1$ (so that the sum of all stencil elements is unity, to avoid damping). We describe our method of choosing these coefficients in Sec. II D below. First, we show how this algorithm leads to perfect dispersion for plane waves on axis in vacuum.

\section{B. Perfect dispersion in vacuum}

To evaluate the effect of the smoothing on the dispersion relation, we compute the smoothing operator in the plane wave basis. If we assume that $\psi \propto e^{-i \mathbf{k} \cdot \mathbf{x}}$, then

$$
\begin{aligned}
S_{x} \psi= & {\left[\alpha_{x}+2 \beta_{y} \cos \left(k_{y} \Delta y\right)+2 \beta_{z} \cos \left(k_{z} \Delta z\right)\right.} \\
& \left.+4 \gamma_{y z} \cos \left(k_{y} \Delta y\right) \cos \left(k_{z} \Delta z\right)\right] \psi,
\end{aligned}
$$

where the $\Delta x_{i}$ are the grid spacings. For convenience we define $s_{i}=\sin ^{2}\left(k_{i} \Delta x_{i} / 2\right)$; then $\cos \left(k_{i} \Delta x_{i}\right)=1-2 s_{i}$. We then have that

$$
\begin{aligned}
S_{x} \psi= & {\left[1-\left(4 \beta_{y}+8 \gamma_{y z}\right) s_{y}-\left(4 \beta_{z}+8 \gamma_{y z}\right) s_{z}\right.} \\
& \left.+16 \gamma_{y z} s_{y} s_{z}\right] \psi .
\end{aligned}
$$

This analysis also shows that plane waves are eigenvectors of the smoothing operators. Since plane waves are also eigenvectors of the derivative operators, each smoothing operator commutes with every derivative operator. Therefore, for a system in vacuum, every field component obeys the modified finite-difference Helmholtz equation,

$$
\frac{1}{c^{2}} D_{t}^{2} \psi=\left(D_{x}^{2} S_{x}+D_{y}^{2} S_{y}+D_{z}^{2} S_{z}\right) \psi
$$

This yields a dispersion relation

$$
\begin{aligned}
\frac{1}{c^{2} \Delta t^{2}} \sin ^{2}\left(\frac{\omega \Delta t}{2}\right)= & \frac{s_{x}}{\Delta x^{2}}\left[1-\left(4 \beta_{y}+8 \gamma_{y z}\right) s_{y}-\left(4 \beta_{z}+8 \gamma_{y z}\right) s_{z}+16 \gamma_{y z} s_{y} s_{z}\right]+\text { cyclic permutations } \\
= & \left(\frac{s_{x}}{\Delta x^{2}}+\frac{s_{y}}{\Delta y^{2}}+\frac{s_{z}}{\Delta z^{2}}\right)-4\left[\left(\frac{\beta_{z}+2 \gamma_{z x}}{\Delta y^{2}}+\frac{\beta_{y}+2 \gamma_{x y}}{\Delta z^{2}}\right) s_{y} s_{z}+\left(\frac{\beta_{x}+2 \gamma_{x y}}{\Delta z^{2}}+\frac{\beta_{z}+2 \gamma_{y z}}{\Delta x^{2}}\right) s_{z} s_{x}\right. \\
& \left.+\left(\frac{\beta_{y}+2 \gamma_{y z}}{\Delta x^{2}}+\frac{\beta_{x}+2 \gamma_{z x}}{\Delta y^{2}}\right) s_{x} s_{y}\right]+16\left(\frac{\gamma_{y z}}{\Delta x^{2}}+\frac{\gamma_{z x}}{\Delta y^{2}}+\frac{\gamma_{x y}}{\Delta z^{2}}\right) s_{x} s_{y} s_{z} .
\end{aligned}
$$

We now choose a distance $\delta$ and define the ratios $r_{i}=\delta^{2} / \Delta x_{i}^{2}$. For convenience we let $s_{i}^{\prime}=r_{i} s_{i}$; then the dispersion relation becomes

$$
\begin{aligned}
& \frac{1}{c^{2} \Delta t^{2}} \sin ^{2}\left(\frac{\omega \Delta t}{2}\right) \\
& =\frac{1}{\delta^{2}}\left\{\left(s_{x}^{\prime}+s_{y}^{\prime}+s_{z}^{\prime}\right)-4\left[\left(\frac{\beta_{z}+2 \gamma_{z x}}{r_{z}}+\frac{\beta_{y}+2 \gamma_{x y}}{r_{y}}\right) s_{y}^{\prime} s_{z}^{\prime}+\left(\frac{\beta_{x}+2 \gamma_{x y}}{r_{x}}+\frac{\beta_{z}+2 \gamma_{y z}}{r_{z}}\right) s_{z}^{\prime} s_{x}^{\prime}+\left(\frac{\beta_{y}+2 \gamma_{y z}}{r_{y}}+\frac{\beta_{x}+2 \gamma_{z x}}{r_{x}}\right) s_{x}^{\prime} s_{y}^{\prime}\right]\right. \\
& \left.\quad+16\left(\frac{\gamma_{y z}}{r_{y} r_{z}}+\frac{\gamma_{z x}}{r_{z} r_{x}}+\frac{\gamma_{x y}}{r_{x} r_{y}}\right) s_{x}^{\prime} s_{y}^{\prime} s_{z}^{\prime}\right\} .
\end{aligned}
$$

We now show through a novel manipulation that this scheme achieves both perfect dispersion and stability. Suppose we choose the $\beta$ and $\gamma$ such that the coefficient of each $s_{i}^{\prime} s_{j}^{\prime}$ term is -1 , and the coefficient of $s_{x}^{\prime} s_{y}^{\prime} s_{z}^{\prime}$ is 1 . In this case, the dispersion relation becomes

$$
\frac{1}{c^{2} \Delta t^{2}} \sin ^{2}\left(\frac{\omega \Delta t}{2}\right)=\frac{1}{\delta^{2}}\left[\left(s_{x}^{\prime}+s_{y}^{\prime}+s_{z}^{\prime}\right)-\left(s_{y}^{\prime} s_{z}^{\prime}+s_{z}^{\prime} s_{x}^{\prime}+s_{x}^{\prime} s_{y}^{\prime}\right)+s_{x}^{\prime} s_{y}^{\prime} s_{z}^{\prime}\right]=\frac{1}{\delta^{2}}\left[1-\left(1-r_{x} s_{x}\right)\left(1-r_{y} s_{y}\right)\left(1-r_{z} s_{z}\right)\right] .
$$


If we choose $\delta \leq \Delta x_{i}$ for each $i$, then $r_{i} \in[0,1]$, and since $s_{i} \in[0,1]$ by definition, $r_{i} s_{i} \in[0,1]$ as well. This implies that $\left(1-r_{i} s_{i}\right) \in[0,1]$ for each $i$, so that

$$
\left[1-\left(1-r_{x} s_{x}\right)\left(1-r_{y} s_{y}\right)\left(1-r_{z} s_{z}\right)\right] \in[0,1] .
$$

Then choosing $c \Delta t \leq \Delta$ we are guaranteed stable propagation. For wave propagation along the $x$ axis, that is, with $k_{y}=k_{z}=0$, the dispersion relation becomes

$$
\frac{1}{c^{2} \Delta t^{2}} \sin ^{2}\left(\frac{\omega \Delta t}{2}\right)=\frac{1}{\delta^{2}} r_{x} s_{x}=\frac{1}{\Delta x^{2}} \sin ^{2}\left(\frac{k_{x} \Delta x}{2}\right) .
$$

For the perfect dispersion relation $\omega / c=k_{x}$ to hold, we then need $c \Delta t=\Delta x$. If $\Delta x \leq \Delta y$ and $\Delta x \leq \Delta z$, we can then choose $\delta=\Delta x$ and achieve stability. Therefore, this algorithm provides perfect dispersion along the axis with the smallest grid spacing as well as stability, while allowing arbitrary cell aspect ratios.

In addition, this algorithm avoids numerical Čerenkov radiation coupled to particles moving relativistically. For a beam propagating in the $x$ direction, we must have a dispersion relation where $\omega / k_{x} \geq c$ for all $\mathbf{k}$ with $\left|k_{i}\right| \leq \pi / \Delta x_{i}$. For this algorithm with the above parameters, we have

$$
\begin{aligned}
\sin ^{2} & \left(\frac{(\omega / c) \Delta x}{2}\right) \\
& =\sin ^{2}\left(\frac{\omega \Delta t}{2}\right) \\
& =1-\left(1-s_{x}\right)\left(1-r_{y} s_{y}\right)\left(1-r_{z} s_{z}\right) \geq 1-\left(1-s_{x}\right) \\
& =\sin ^{2}\left(\frac{k_{x} \Delta x}{2}\right)
\end{aligned}
$$

and thus $\omega / k_{x} \geq c$.

\section{Divergence relations}

The controlled dispersion equations (1) still conserve electric charge, as $D_{t}(\boldsymbol{\nabla} \cdot \mathbf{E})=-(\boldsymbol{\nabla} \cdot \mathbf{J}) / \epsilon_{0}$. However, the condition of zero numerical divergence of $\mathbf{B}$ does not necessarily hold. Instead, we have the modified condition $\boldsymbol{\nabla}^{\prime} \cdot \mathbf{B}=0$, which can be shown by

$$
D_{t}\left(\boldsymbol{\nabla}^{\prime} \cdot \mathbf{B}\right)=\nabla^{\prime} \cdot D_{t} \mathbf{B}=-\nabla^{\prime} \cdot\left(\boldsymbol{\nabla}^{\prime} \times \mathbf{E}\right)=0 .
$$

This follows from the commutativity of the $S$ and $D$ operators.

\section{Choice of coefficients}

We now describe our method of choosing the smoothing coefficients. The conditions on the coefficients of the $s_{i}^{\prime}$ products in Eq. (7) require

$$
4\left(\frac{\beta_{z}+2 \gamma_{z x}}{r_{z}}+\frac{\beta_{y}+2 \gamma_{x y}}{r_{y}}\right)=1,
$$

$$
\begin{gathered}
4\left(\frac{\beta_{x}+2 \gamma_{x y}}{r_{x}}+\frac{\beta_{z}+2 \gamma_{y z}}{r_{z}}\right)=1, \\
4\left(\frac{\beta_{y}+2 \gamma_{y z}}{r_{y}}+\frac{\beta_{x}+2 \gamma_{z x}}{r_{x}}\right)=1, \\
16\left(\frac{\gamma_{y z}}{r_{y} r_{z}}+\frac{\gamma_{z x}}{r_{z} r_{x}}+\frac{\gamma_{x y}}{r_{x} r_{y}}\right)=1 .
\end{gathered}
$$

This is four equations in six unknowns; however, we can determine the $\beta$ and $\gamma$ uniquely if we impose the condition that $\beta_{i}=\beta^{\prime} r_{i}$ for each $i$ and some parameter $\beta^{\prime}$. This is motivated by the desire to have smoothing along a direction be reduced as the grid spacing in that direction is increased; this implies $\beta_{i} \rightarrow 0$ as $r_{i} \rightarrow 0$. We then have

$$
4\left(2 \beta^{\prime}+\frac{2 \gamma_{z x}}{r_{z}}+\frac{2 \gamma_{x y}}{r_{y}}\right)=1,
$$

so that

$$
\frac{1}{8}-\beta^{\prime}=\frac{\gamma_{z x}}{r_{z}}+\frac{\gamma_{x y}}{r_{y}}=r_{x}\left(\frac{\gamma_{z x}}{r_{z} r_{x}}+\frac{\gamma_{x y}}{r_{x} r_{y}}\right) .
$$

Then, summing over cyclic permutations and using Eq. (16), we have

$$
\left(\frac{1}{r_{x}}+\frac{1}{r_{y}}+\frac{1}{r_{z}}\right)\left(\frac{1}{8}-\beta^{\prime}\right)=\frac{1}{8},
$$

and thus

$$
\beta^{\prime}=\frac{1}{8}\left[1-\left(\frac{1}{r_{x}}+\frac{1}{r_{y}}+\frac{1}{r_{z}}\right)^{-1}\right] .
$$

Our coefficients are then

$$
\begin{gathered}
\beta_{x}=\frac{r_{x}}{8}\left(1-\frac{r_{x} r_{y} r_{z}}{r_{y} r_{z}+r_{z} r_{x}+r_{x} r_{y}}\right), \\
\gamma_{y z}=r_{y} r_{z}\left(\frac{1}{16}-\frac{1}{8} \frac{r_{y} r_{z}}{r_{y} r_{z}+r_{z} r_{x}+r_{x} r_{y}}\right),
\end{gathered}
$$

and cyclic permutations.

\section{E. Choice of numerical parameters in a plasma}

We have seen in Sec. II B that perfect dispersion for plane waves in vacuum in the $x$ direction can be obtained by setting $c \Delta t=\Delta x$. However, in practice $c \Delta t$ is typically chosen to be slightly less than the analytic limit to guarantee stability in the presence of roundoff error. At the same time, the analytic group velocity in a plasma is slightly less than $c$. As it turns out, we can choose $c \Delta t$ that is both stable in this algorithm and that guarantees the correct group velocity along an axis in a plasma at a given frequency. We showed above that the dispersion relation for plane waves propagating in the $x$ direction in the controlled dispersion algorithm is the same as that of the 
standard Yee algorithm. We can therefore expect the dispersion relations in a plasma to be the same. Adapting the formula from [27], we have

$$
\frac{4}{c^{2} \Delta t^{2}} \sin ^{2}\left(\frac{\omega \Delta t}{2}\right)=\frac{4}{\Delta x^{2}} \sin ^{2}\left(\frac{k_{x} \Delta x}{2}\right)+k_{p}^{2}
$$

where $k_{p}$ is the plasma wave number.

We can compute the group velocity by taking derivatives with respect to $k_{x}$. Letting $\beta_{g}=v_{g} / c$, this gives

$$
\beta_{g}=\frac{2 c \Delta t}{\Delta x} \frac{1}{\sin (\omega \Delta t)} \sin \left(\frac{k_{x} \Delta x}{2}\right) \cos \left(\frac{k_{x} \Delta x}{2}\right) .
$$

Now, typically the frequency is fixed, and the resulting wave numbers depend on the physical system. We therefore represent terms with $k_{x}$ in terms of $\omega$, using Eq. (23). We have

$$
\begin{aligned}
\beta_{g}= & \frac{2 c \Delta t}{\Delta x} \frac{1}{\sin (\omega \Delta t)}\left[\frac{\Delta x^{2}}{c^{2} \Delta t^{2}} \sin ^{2}\left(\frac{\omega \Delta t}{2}\right)-\frac{k_{p}^{2} \Delta x^{2}}{4}\right]^{1 / 2} \\
& \times\left[1-\frac{\Delta x^{2}}{c^{2} \Delta t^{2}} \sin ^{2}\left(\frac{\omega \Delta t}{2}\right)+\frac{k_{p}^{2} \Delta x^{2}}{4}\right]^{1 / 2} .
\end{aligned}
$$

Thus, given $k_{p}, \omega$, and $\Delta x$, we can numerically solve for the $\Delta t$ such that the $\beta_{g}$ in Eq. (25) equals the analytic value of $\sqrt{1-k_{p}^{2} c^{2} / \omega^{2}}$. While this choice of $\Delta t$ is optimized for the 1D linear case, we find that it provides excellent group velocity accuracy in the channeled and nonlinear cases as well, which we demonstrate as we employ this method for the tests in the following sections.

\section{LINEAR GROUP VELOCITY TESTS}

As a basic test of the controlled dispersion algorithm, we compute the group velocity of electromagnetic waves along an axis and compare to the standard Yee case. Our test case consists of 2D simulations of a linear $\left(a_{0}=10^{-3}\right)$ laser pulse in a matched parabolic plasma channel. For linear pulses, the group velocity can be computed analytically, so we can compare both normal and controlled dispersion simulations to theoretical values. It is known that a Gaussian laser mode with spot size $w_{0}$ is matched to a plasma channel with transverse profile $n=$ $n_{0}+r^{2} / \pi r_{e} w_{0}^{4}$ [36], where $w_{0}$ is that waist size of a Gaussian beam as per [37]. As our figure of merit, we use the dephasing factor

$$
f_{d}=\frac{1}{\beta_{g}}-1,
$$

with $\beta_{g}=v_{g} / c$, which describes the normalized rate at which a laser pulse slips behind a highly relativistic beam. This figure of merit was introduced and discussed in greater detail in [38].

We run tests for a range of plasma densities $n_{0}$ at the bottom of the channel. For each $n_{0}$, we let $\lambda_{p}=2 \pi / k_{p}^{(0)}$ be the corresponding plasma wavelength, so that $k_{p}^{(0)}=$ $\sqrt{4 \pi n_{0} r_{e}}$. Then we set the rms length $L_{\mathrm{rms}}$ of the Gaussian laser pulse intensity such that $k_{p}^{(0)} L_{\mathrm{rms}}=1$, and the transverse waist size such that $w_{0}=\lambda_{p}$. We propagate the laser pulse up a cosine density ramp of length $10 \mu \mathrm{m}$, focusing the spot at the start of the longitudinally uniform density region. The laser pulse has a center wavelength of $\lambda=800 \mathrm{~nm}$. We use grid spacings of $\Delta x=\lambda / 24$ and $\Delta y=\lambda / 3$, typical of explicit LPA simulations. For the normal dispersion simulations, we set $\Delta t$ to be close to its Courant limit, while for the controlled dispersion simulations, we choose $\Delta t$ based on the computation in Sec. IIE.

The results of these tests for a range of densities, along with the analytic values, are shown in Fig. 1. Here the computation of the analytic values include the channel effects, following the formula in [38]. We find that both algorithms show very little dephasing error at high density but that the error for normal dispersion increases as the density decreases. However, the controlled dispersion algorithm maintains group velocity accuracy throughout the density range. At the lowest density, the dephasing factor with controlled dispersion is only $1.7 \%$ greater than the analytic value, while for standard Yee the dephasing factor is 4.7 times the analytic value; this represents a factor of 280 improvement with controlled dispersion. This shows that the calculation of $\Delta t$ in Sec. II E, based on plane wave propagation in a uniform plasma, is sufficient for the case of a channel-guided pulse. The lower relative error at higher density is due to the fact that the physical dephasing from the plasma and channel is larger and dominates the numerical dispersion.

While it is not necessary to use the same aspect ratio at any density, the controlled dispersion algorithm still has an advantage even when this constraint is lifted for normal

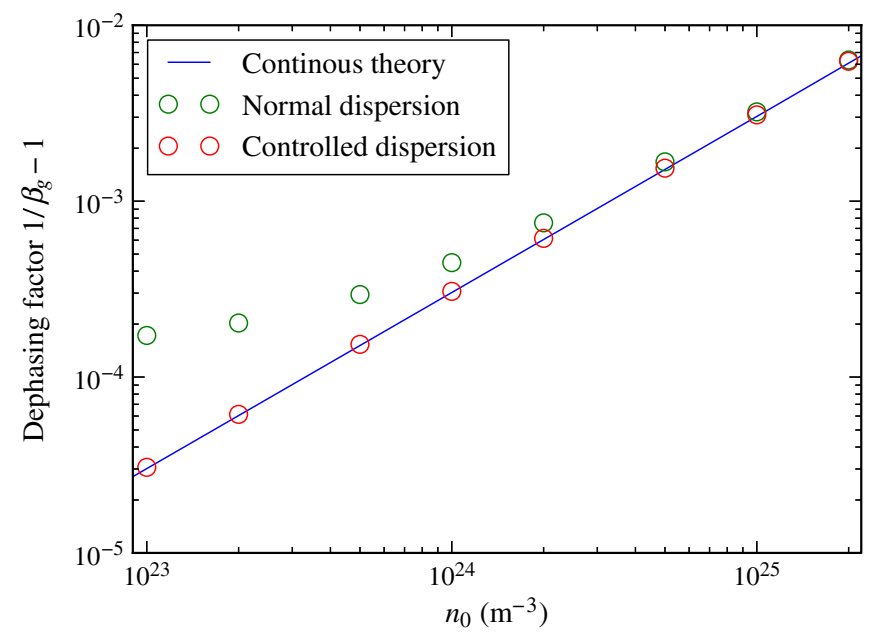

FIG. 1. The dephasing factors for normal dispersion and controlled dispersion simulations compared to continuous theory. $n_{0}$ is the plasma density at the bottom of the plasma channel. We plot the dephasing factor here for consistency with a similar analysis of the envelope model in [38]. 
dispersion simulations. Rather than using $\Delta y=\lambda / 3$, one could scale the transverse grid spacing proportionally to the plasma wavelength. This increases the aspect ratio and hence decreases the dephasing error. However, it was shown in [38] that with normal dispersion, the dephasing error is still over $30 \%$ at the lowest density. In any event, certain cases, such as bubble regime simulations or the modeling of a trapped bunch, require higher transverse resolution than that needed simply to resolve the plasma wavelength. Since increasing the longitudinal resolution is computationally impractical, this mandates a lower aspect ratio than $\lambda_{p} / \lambda$, and the advantage of controlled dispersion is manifest [39].

\section{IMPLICATIONS FOR SIMULATIONS OF LPA STAGES}

Dephasing plays a key role in the design of stages for a high-energy laser-plasma collider [9]. We therefore expect that correct dispersion will be an important aspect of the simulation of such stages. We thus proceed to test the effects of numerical dispersion on simulations of a candidate stage design. Our test case is a meter-scale, quasilinear laser wakefield accelerator stage proposed for development at the BELLA facility at Lawrence Berkeley National Laboratory [40]. In the quasilinear regime, the laser intensity has $a_{0} \approx 1$ to allow transverse focusing of both electron and positron beams. This is well below the threshold for self-trapping, so these stages are designed for an externally injected bunch.

For this case, we take the stage to consist of a plasma channel which is longitudinally uniform and transversely parabolic, with a density of $10^{23} \mathrm{~m}^{-3}$ on axis. This corresponds to a plasma wavelength of $106 \mu \mathrm{m}$; then the onedimensional linear dephasing length, over which a plane wave pulse would slip in phase by $\pi$ relative to a speed-oflight particle bunch, is $1.84 \mathrm{~m}$. Since performing such meter-scale simulations with explicit, laboratory-frame FDTD is intractable, even in 2D, we scale the simulation to higher density $[40,41]$ to reduce the simulation size. While performing the simulation in a Lorentz boosted frame is another possibility for reducing the computational cost, we perform the test in the laboratory frame for several reasons. First, the laboratory frame is better suited to simulations that include particle injection, so benchmarking the algorithm in that frame is important. Second, controlled dispersion (with cubic cells) has been tested, and indeed shown to be essential, in boosted-frame simulations [31].

For our tests we scale the on-axis density to $n_{0}=$ $10^{24} \mathrm{~m}^{-3}$, reducing laser pulse length and spot size by a factor of $\sqrt{10}$. The plasma wavelength corresponding to the on-axis density is therefore $\lambda_{p}=33.4 \mu \mathrm{m}$; we use an rms (intensity) matched laser pulse length of $L_{\mathrm{rms}}=\lambda_{p} / 2 \pi=$ $5.31 \mu \mathrm{m}$, and a transverse spot size $w_{0}$ (defined as per [37]) given by $k_{p} w_{0}=5.3$ based on the considerations in
[40]. The peak normalized laser pulse intensity is given by $a_{0}=1$ and the central wavelength is $\lambda_{0}=800 \mathrm{~nm}$.

We run 2D simulations using both the normal dispersion and controlled dispersion algorithms. In each case we use a longitudinal grid spacing of $\Delta x=\lambda_{0} / 16=50 \mathrm{~nm}$ and transverse grid spacing of $\Delta y=\lambda_{0} / 2=400 \mathrm{~nm}$. This low longitudinal resolution is chosen to conserve computational resources, a key consideration in LPA stage simulations. Our simulation domain sizes are $L_{x}=117 \mu \mathrm{m}$ and $L_{y}=181 \mu \mathrm{m}$, for a grid size of $2338 \times 452$. We test our methods by introducing test electrons at the beginning of the simulation and observing their dynamics. The test particles have negligible charge and mass, so they follow trajectories of physical electrons but do not deposit current or otherwise affect the fields. We initialize them in a rectangular region $\lambda_{p}$ long by $0.02 \lambda_{p}=668 \mathrm{~nm}$ wide, centered $1.625 \lambda_{p}$ behind the peak of the laser pulse longitudinally (in the second plasma bucket) and on axis transversely. They are given an initial energy of $1.5 \gamma_{g} m c^{2}=31 \mathrm{MeV}$, with $\gamma_{g}=\left(1-\beta_{g}^{2}\right)^{-1 / 2}$, and no transverse momentum. We propagate the laser pulse and particles for $82 \mathrm{~mm}$, about 1.4 times the 1D linear, scaled dephasing length. In the controlled dispersion simulation, the smoothing coefficients were $\alpha_{x}=0.9960, \alpha_{y}=0.7501, \beta_{x}=0.1250$, and $\beta_{y}=$ 0.0020 . Since this was a $2 \mathrm{D}$ simulation, the remaining coefficients were not used.

The differences between the normal dispersion and controlled dispersion algorithms are stark. At each data dump, we find the maximum longitudinal momentum of the test particles. This is plotted for the two algorithms in Fig. 2. We find that the particles in the controlled dispersion simulation achieve $64 \%$ greater maximum momentum

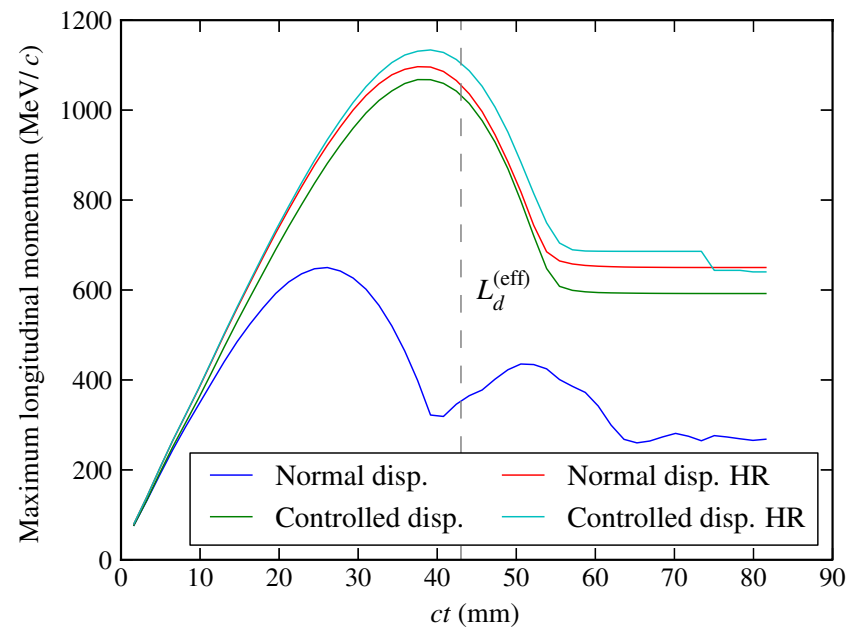

FIG. 2. The maximum test particle momentum, as a function of normalized propagation time, which is approximately equal to propagation distance. The dashed line indicates the effective linear dephasing length, $L_{d}=43.0 \mathrm{~mm}$. In the legend, "HR" refers to the high resolution simulations, with $\Delta x=\lambda_{0} / 32$; those not labeled HR have $\Delta x=\lambda_{0} / 16$. 

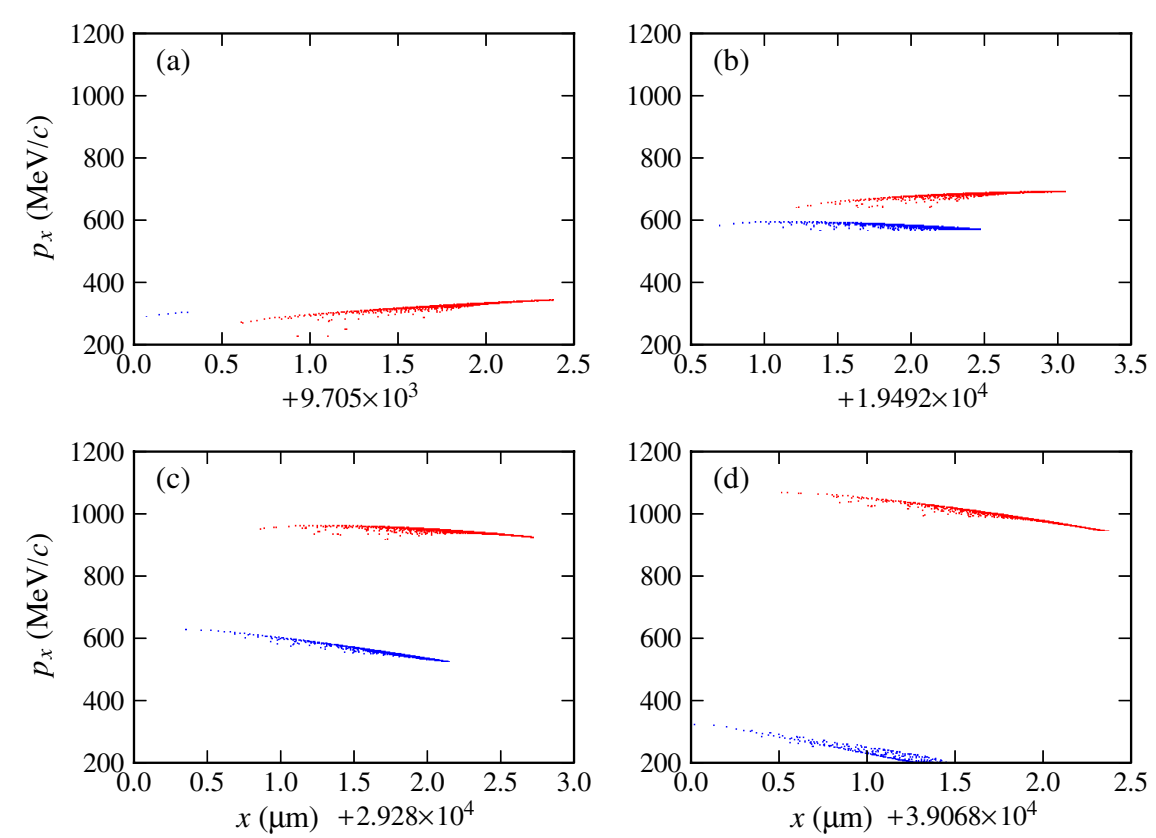

FIG. 3. Longitudinal phase space of the particles, at (a) $9.7 \mathrm{~mm}$, (b) $19.5 \mathrm{~mm}$, (c) $29.3 \mathrm{~mm}$, and (d) $39.1 \mathrm{~mm}$ of propagation. The normal dispersion case is shown in blue and controlled dispersion in red.

than those in the normal dispersion case, and reach that maximum after an acceleration distance $44 \%$ greater. In fact, in the controlled dispersion simulation, the maximum momentum is reached in a distance quite close to the linear dephasing length. To make this comparison, we must take into account the requirement that particles remain in the transverse focusing phase in addition to the accelerating phase, as only such particles are effectively accelerated. Since the length of the combined accelerating and focusing phase is not analytically simple in the quasilinear regime [42], we determine it numerically. We inspect both the accelerating field $E_{x}$ and the focusing gradient $\partial E_{y} / \partial y$ on axis near the beginning of the simulation, before nonlinear effects have altered the laser pulse and hence the plasma wake. We find that the length of the combined accelerating and focusing phase is $\Lambda=0.40 \lambda_{p}$. The effective dephasing length is then the distance over which the laser pulse slips by $\Lambda$ relative to a speed-of-light particle bunch; with the linear group velocity, including channeling effects, this gives $L_{d}^{(\text {eff })}=43.0 \mathrm{~mm}$. The maximum momentum is reached at close to this propagation distance in the controlled dispersion simulation, but at a much shorter distance with normal dispersion. As an additional check, we repeat both simulations with double the longitudinal resolution, namely $\Delta x=\lambda_{0} / 32$. We find that the low resolution controlled dispersion simulation closely reproduces the results of both high resolution simulations, while the low resolution normal dispersion simulation differs significantly. The controlled dispersion algorithm achieves this accuracy at much less computational cost than simply increasing the resolution: Increasing the resolution increases the cost by roughly a factor of 4 through doubling both the longitudinal grid size and the number of time steps. Controlled dispersion, on the other hand, just increases the stencil size of one of the electromagnetic field updates. Since the computational time in PIC simulations is typically dominated by the particle advance, this results in only a modest increase in cost of each step; in fact we observed that this was more than balanced by the reduction in the number of time steps allowed by the larger time step.

We also show, in Fig. 3, the evolution of the longitudinal phase space of a test particle bunch. The test particle bunch was selected from all the test particles in the simulation by examining the data at the time the maximum energy was achieved. We first selected the particles which at that time had at least $90 \%$ of the maximum energy, and then computed the initial longitudinal phases of those particles. All particles in that interval of initial longitudinal phase were then selected as the test bunch and plotted in the figure. We can see that phase space rotation, and subsequent dephasing, proceeds faster in the normal dispersion case. Thus, the numerical dispersion correction available with the controlled dispersion algorithm allows significantly more accurate LPA stage simulations.

\section{BEAM INITIALIZATION}

Many accelerator simulations involve the interaction of an externally injected beam with an accelerator component. For laser-plasma acceleration, this is true for simulations of subsequent stages in an LPA collider; apart from LPA, externally injected beams also appear in simulations of wakefields in structure-based accelerators. For such simulations, it is essential to initialize the beam self-fields 
consistently as the beam particles are loaded. Loading a beam with no fields or incorrect fields leads to unphysical transition radiation. This issue is sometimes addressed by propagating the beam for a long distance in the pipe before the interaction to allow the fields to stabilize. However, if the controlled dispersion algorithm is used to avoid numerical Čerenkov radiation, that approach becomes more difficult: Since controlled dispersion allows fields to propagate with group velocity equal to $c$, the relative velocity difference between the fields and the beam becomes $1-$ $\beta \approx 1 / 2 \gamma^{2}$. For the fields to stabilize then requires a propagation distance of $\sim 2 \gamma^{2} \sigma_{x}$, where $\sigma_{x}$ is the bunch length, which becomes prohibitive for highly relativistic beams, and could be even longer than the acceleration length in an LPA stage.

We can use the controlled dispersion stencil to implement an initialization procedure that both solves for the correct beam fields initially, and propagates those fields consistently to avoid both unphysical transition radiation or numerical Čerenkov radiation. We compute the initial fields by first solving for the potentials, and then take the appropriate derivatives to find the fields. However, we must be careful to choose consistent smoothed or unsmoothed derivatives. If we define the potentials by
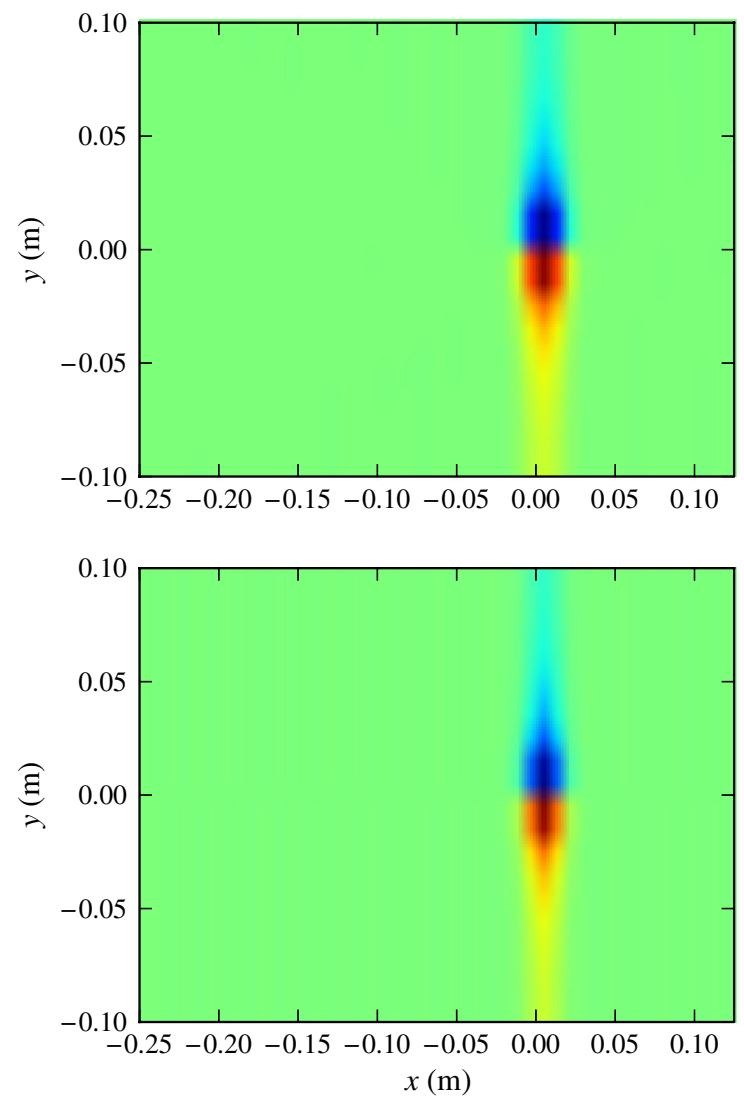

$$
\mathbf{E}=-\nabla^{\prime} \Phi-D_{t} \mathbf{A}, \quad \mathbf{B}=\boldsymbol{\nabla}^{\prime} \times \mathbf{A},
$$

then the controlled dispersion discrete Maxwell equations in Eq. (1) hold. We also impose the Lorenz gauge condition,

$$
\frac{1}{c^{2}} D_{t} \Phi+\boldsymbol{\nabla} \cdot \mathbf{A}=0
$$

as well as the stationary condition that for any field or potential component $\psi, D_{t} \psi=-\beta c D_{x} \psi$. Combining these relations, we obtain the second order differential equations

$$
\begin{aligned}
& \left(\nabla^{\prime} \cdot \boldsymbol{\nabla}-\beta^{2} D_{x}^{2}\right) \Phi=-\frac{\rho}{\epsilon_{0}}, \\
& \left(\nabla^{\prime} \cdot \boldsymbol{\nabla}-\beta^{2} D_{x}^{2}\right) \mathbf{A}=-\mu_{0} \mathbf{J}
\end{aligned}
$$

for the potentials, and relations for the fields

$$
\mathbf{E}=-\nabla^{\prime} \Phi+\hat{\mathbf{x}} \beta^{2} D_{x} \Phi, \quad \mathbf{B}=\boldsymbol{\nabla}^{\prime} \times \mathbf{A} .
$$

We compute the potentials using an implicit linear solve, and then use a finite difference to compute the initial fields. We note that the operator in Eq. (29) is symmetric, since we can write
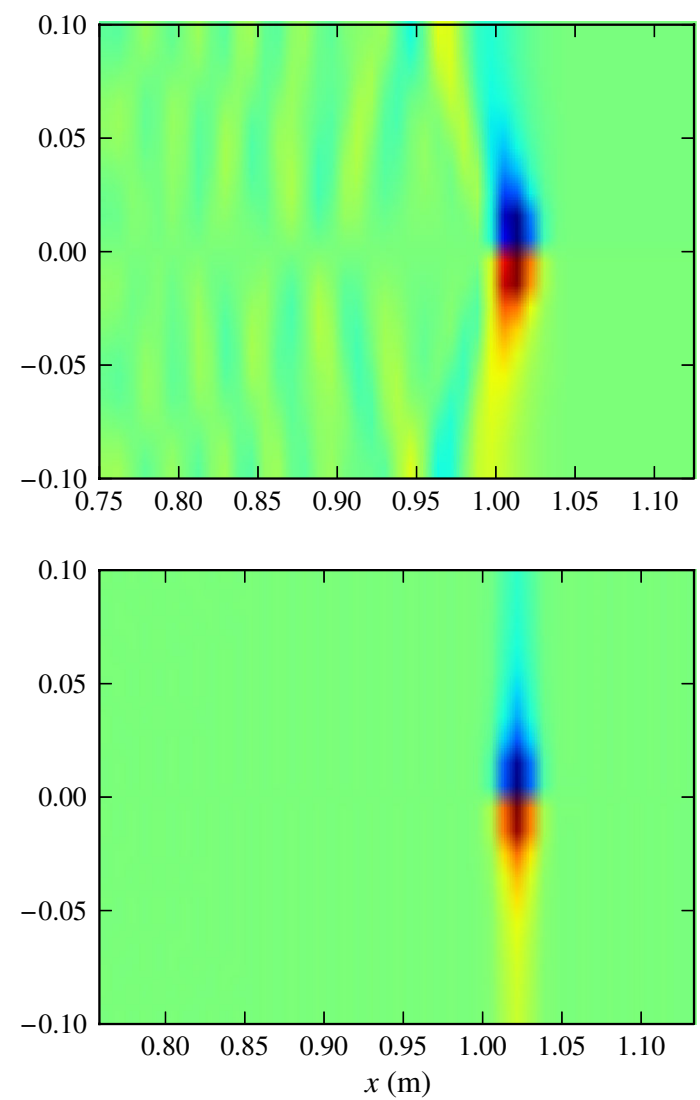

FIG. 4. Transverse field $E_{y}$ at the pipe midplane in $z$. Top left: Initial field with the normal dispersion algorithm; bottom left: initial field with the controlled dispersion algorithm; top right: field after $1 \mathrm{~m}$ propagation with normal dispersion; bottom right: field after propagation with controlled dispersion. 


$$
\nabla^{\prime} \cdot \boldsymbol{\nabla}=D_{x}^{T} S_{x} D_{x}+D_{y}^{T} S_{y} D_{y}+D_{z}^{T} S_{z} D_{z},
$$

where the $D_{i}$ are the forward difference operators.

We test this procedure by initializing and propagating an electron bunch and its fields in a smooth, rectangular, perfectly conducting pipe. The pipe is $20 \mathrm{~cm}$ on each side. We use a $1 \mathrm{nC}$ Gaussian electron bunch with longitudinal rms length $\sigma_{x}=5 \mathrm{~mm}$ and transverse rms size $\sigma_{r}=1 \mathrm{~mm}$. The bunch is cold, with uniform energy of $100 \mathrm{MeV}$ and no transverse momentum. In the simulation, we use longitudinal grid spacing $\Delta x=8.33 \mathrm{~mm}$ and transverse grid spacing $\Delta y=\Delta z=10 \mathrm{~mm}$. We note that the bunch is poorly resolved on this grid; this is done to reflect a common condition in accelerator simulations, where the bunch is much smaller than the surrounding structure and one wishes to avoid increasing the resolution to highly resolve the beam. In such cases, this can still provide adequate resolution of the beam wakefield, which is the object of the simulation, while self-consistent beam dynamics is less important. We propagate the beam for $1 \mathrm{~m}$.

For comparison, we perform the same initialization and propagation procedure using the standard Yee algorithm, by taking $\boldsymbol{\nabla}^{\prime} \rightarrow \boldsymbol{\nabla}$. The equations for the potentials and fields, analogous to Eqs. (29) and (30), become

$$
\begin{aligned}
\left(\frac{1}{\gamma^{2}} D_{x}^{2}+\nabla_{\perp}^{2}\right) \Phi & =-\frac{\rho}{\epsilon_{0}}, \\
\left(\frac{1}{\gamma^{2}} D_{x}^{2}+\nabla_{\perp}^{2}\right) \mathbf{A} & =-\mu_{0} \mathbf{J}
\end{aligned}
$$

and

$$
\mathbf{E}=-\frac{1}{\gamma^{2}} D_{x} \Phi-\nabla_{\perp} \Phi, \quad \mathbf{B}=\boldsymbol{\nabla} \times \mathbf{A}
$$

The results of these tests are shown in Fig. 4. The figure shows the transverse field $E_{y}$ at the $x y$ cross section of the pipe located at the middle of the pipe in $z$. The plots on the left show the initial field, and those on the right show the field after $1 \mathrm{~m}$ of propagation; the top row of plots shows the normal dispersion algorithm and the bottom row controlled dispersion. We see that the initial fields look similar for the two algorithms, but as the beam propagates, numerical dispersion in the normal dispersion case causes the transverse fields to curve backward away from the beam. Physically, the pattern should be longitudinally symmetric around the beam location, and at $100 \mathrm{MeV}$ beam energy the longitudinal extent of the field pattern should be negligible. The shape of the fields suggests that energy is propagating transversely outward at a slight angle, consistent with the presence of numerical Čerenkov radiation. With the controlled dispersion update, the fields are unchanged as expected.

\section{CONCLUSION}

We have described in detail a modified finite-difference time-domain electromagnetic algorithm which provides numerical dispersion-free propagation along an axis. This algorithm allows a semi-infinite range of cell aspect ratios, while being fully explicit. We demonstrated the improvement over the standard Yee algorithm for the test case of a linear laser pulse in a plasma channel, and then showed the benefit of the algorithm in the application to high-energy, quasilinear LPA stage simulation. Finally, we showed how the controlled dispersion stencil can be used for consistent initialization of a relativistic particle bunch, without unphysical transition or Čerenkov radiation. Thus, the controlled dispersion algorithm described here can benefit accelerator simulation in a wide variety of cases.

\section{ACKNOWLEDGMENTS}

This work was supported by U.S. Department of Energy, Office of Science Grants No. DE-FC02-07ER41499 (SciDAC), No. DE-SC0000845 (SBIR), and No. DEAC02-05CH11231 (LBNL); and by Tech-X Corporation. This research used resources of the National Energy Research Scientific Computing Center, which is supported by the Office of Science of the U.S. Department of Energy under Contract No. DE-AC02-05CH11231. In addition, we acknowledge additional support for VORPAL development from the offices of Fusion Energy Sciences, High Energy Physics, and Nuclear Physics and the SciDAC program of the Department of Energy Office of Science, the Air Force Office of Scientific Research, the Joint Technology Office, and Office of the Secretary of Defense, and the SBIR programs of the Department of Energy and Department of Defense. The authors would like to thank J.-L. Vay, B. A. Shadwick, S. Y. Kalmykov, C. B. Schroeder, E. Esarey, and W. P. Leemans for helpful discussions and advice. We also acknowledge members of the VORPAL development team: D. Alexander, K. Amyx, E. Angle, T. Austin, G. I. Bell, Y. Choi, R. K. Crockett, D. A. Dimitrov, M. Durant, B. Jamroz, M. Koch, S. E. Kruger, A. Likhanskii, M. C. Lin, M. Loh, J. Loverich, S. Mahalingam, P. J. Mullowney, C. Nieter, K. Paul, I. Pogorelov, C. Roark, D. Robertson, B. T. Schwartz, S. W. Sides, D. N. Smithe, P. H. Stoltz, S. A. Veitzer, D. J. Wade-Stein, G. R. Werner, N. Xiang, and C. D. Zhou.

[1] Allen Taflove and Susan C. Hagness, Computational Electrodynamics: The Finite-Difference Time-Domain Method (Artech House, Boston, 2005), 3rd ed.

[2] K. S. Yee, IEEE Trans. Antennas Propag. 14, 302 (1966).

[3] Igor Zagorodnov, Rolf Schuhmann, and Thomas Weiland, J. Comput. Phys. 191, 525 (2003).

[4] T. Tajima and J.M. Dawson, Phys. Rev. Lett. 43, 267 (1979).

[5] E. Esarey, P. Sprangle, J. Krall, and A. Ting, IEEE Trans. Plasma Sci. 24, 252 (1996).

[6] E. Esarey, C. B. Schroeder, and W. P. Leemans, Rev. Mod. Phys. 81, 1229 (2009).

[7] C. D. Decker and W. B. Mori, Phys. Rev. Lett. 72, 490 (1994). 
[8] C. B. Schroeder, C. Benedetti, E. Esarey, and W. P. Leemans, Phys. Rev. Lett. 106, 135002 (2011).

[9] C. B. Schroeder, E. Esarey, C. G. R. Geddes, C. Benedetti, and W.P. Leemans, Phys. Rev. ST Accel. Beams 13, 101301 (2010).

[10] Patrick Mora and Thomas M. Antonsen, Jr., Phys. Plasmas 4, 217 (1997).

[11] D. F. Gordon, W. B. Mori, and T. M. Antonsen, Jr., IEEE Trans. Plasma Sci. 28, 1135 (2000).

[12] R. F. Hubbard, D. Kaganovich, B. Hafizi, C. I. Moore, P. Sprangle, A. Ting, and A. Zigler, Phys. Rev. E 63, 036502 (2001).

[13] C. G. R. Geddes, Cs. Toth, J. van Tilborg, E. Esarey, C. B. Schroeder, D. L. Bruhwiler, C. Nieter, J. R. Cary, and W. P. Leemans, Nature (London) 431, 538 (2004).

[14] S.P.D. Mangles, C.D. Murphy, Z. Najmudin, A. G. R. Thomas, J. L. Collier, A.E. Dangor, E. J. Divall, P.S. Foster, J. G. Gallacher, C. J. Hooker, D. A. Jaroszynski, A. J. Langley, W. B. Mori, P. A. Norreys, F. S. Tsung, R. Viskup, B. R. Walton, and K. Krushelnick, Nature (London) 431, 535 (2004).

[15] J. Faure, Y. Glinec, A. Pukhov, S. Kiselev, S. Gordienko, E. Lefebvre, J.-P. Rousseau, F. Burgy, and V. Malka, Nature (London) 431, 541 (2004).

[16] F. V. Hartemann, D. J. Gibson, W. J. Brown, A. Rousse, K. Ta Phuoc, V. Malka, J. Faure, and A. Pukhov, Phys. Rev. ST Accel. Beams 10, 011301 (2007).

[17] F. Grüner, S. Becker, U. Schramm, T. Eichner, M. Fuchs, R. Weingartner, D. Habs, J. Meyer-ter-Vehn, M. Geissler, M. Ferrario, L. Serafini, B. van der Geer, H. Backe, W. Lauth, and S. Reiche, Appl. Phys. B 86, 431 (2007).

[18] C. B. Schroeder, W. M. Fawley, E. Esarey, and W. P. Leemans, in Proceedings of the 28th International Free Electron Laser Conference, Berlin, Germany, 2006 (JACoW, 2006), pp. 455-458 [http://www.jacow.org].

[19] D. A. Jaroszynski, R. Bingham, E. Brunetti, B. Ersfeld, J. Gallacher, B. van der Geer, R. Issac, S. P. Jamison, D. Jones, M. de Loos, A. Lyachev, V. Pavlov, A. Reitsma, Y. Saveliev, G. Vieux, and S. M. Wiggins, Phil. Trans. R. Soc. A 364, 689 (2006).

[20] W. P. Leemans, B. Nagler, A. J. Gonsalves, Cs. Toth, K. Nakamura, C. G. R. Geddes, E. Esarey, C. B. Schroeder, and S. M. Hooker, Nat. Phys. 2, 696 (2006).

[21] C. G. R. Geddes, K. Nakamura, G. R. Plateau, Cs. Toth, E. Cormier-Michel, E. Esarey, C. B. Schroeder, J. R. Cary, and W. P. Leemans, Phys. Rev. Lett. 100, 215004 (2008).

[22] A. J. Gonsalves, K. Nakamura, C. Lin, D. Panasenko, S. Shiraishi, T. Sokollik, C. Benedetti, C. B. Schroeder, C. G. R. Geddes, J. van Tilborg, J. Osterhoff, E. Esarey, C. Toth, and W.P. Leemans, Nat. Phys. 7, 862 (2011).

[23] J. Faure, C. Rechatin, A. Norlin, A. Lifschitz, Y. Glinec, and V. Malka, Nature (London) 444, 737 (2006).
[24] C. K. Birdsall and A. B. Langdon, Plasma Physics via Computer Simulation (McGraw-Hill, New York, 1985).

[25] R. W. Hockney and J. W. Eastwood, Computer Simulation Using Particles (McGraw-Hill, New York, 1981).

[26] A. Pukhov and J. Meyer-ter-Vehn, Appl. Phys. B 74, 355 (2002).

[27] F. S. Tsung, W. Lu, M. Tzoufras, W. B. Mori, C. Joshi, J. M. Vieira, L. O. Silva, and R. A. Fonseca, Phys. Plasmas 13, 056708 (2006).

[28] W. Lu, M. Tzoufras, C. Joshi, F. S. Tsung, W. B. Mori, J. Vieira, R. A. Fonseca, and L. O. Silva, Phys. Rev. ST Accel. Beams 10, 061301 (2007).

[29] S. Kalmykov, S. A. Yi, V. Khudik, and G. Shvets, Phys. Rev. Lett. 103, 135004 (2009).

[30] S. Y. Kalmykov et al., New J. Phys. 12, 045019 (2010).

[31] J.-L. Vay, C. G. R. Geddes, E. Cormier-Michel, and D. P. Grote, J. Comput. Phys. 230, 5908 (2011).

[32] J.S. Juntunen and T.D. Tsiboukis, IEEE Trans. Microwave Theory Tech. 48, 582 (2000).

[33] Igor Zagorodnov and Thomas Weiland, Phys. Rev. ST Accel. Beams 8, 042001 (2005).

[34] A. J. Pukhov, J. Plasma Phys. 61, 425 (1999).

[35] Mikko Kärkkäinen, Erion Gjonaj, Thomas Lau, and Thomas Weiland, in Proceedings of the 2006 International Computational Accelerator Physics Conference, Chamonix, France, 2006 (JACoW, 2006), pp. 35-40 [http://www.jacow.org].

[36] E. Esarey, P. Sprangle, J. Krall, and A. Ting, IEEE J. Quantum Electron. 33, 1879 (1997).

[37] A. E. Siegman, Lasers (University Science Books, Mill Valley, CA, 1986).

[38] Benjamin M. Cowan, David L. Bruhwiler, Estelle Cormier-Michel, Eric Esarey, Cameron G. R. Geddes, Peter Messmer, and Kevin M. Paul, J. Comput. Phys. 230, 61 (2011).

[39] B. M. Cowan, S. Y. Kalmykov, A. Beck, X. Davoine, K. Bunkers, A. F. Lifschitz, E. Lefebvre, D. L. Bruhwiler, B. A. Shadwick, and D. P. Umstadter, J. Plasma Phys. 78, 469 (2012).

[40] C. G. R. Geddes, E. Cormier-Michel, E. Esarey, C. B. Schroeder, W. P. Leemans, D. L. Bruhwiler, B. Cowan, C. Nieter, K. Paul, and J. R. Cary, in Proceedings of the 2009 Particle Accelerator Conference, Vancouver, BC, Canada, 2009 (JACoW, 2010), p. 2970 [http://www.jacow.org].

[41] E. Cormier-Michel, C. G. R. Geddes, E. Esarey, C. B. Schroeder, D. L. Bruhwiler, K. Paul, B. Cowan, and W.P. Leemans, in Thirteenth Advanced Accelerator Concepts, Santa Cruz, CA, 2008, edited by C.B. Schroeder, W.P. Leemans, and E. Esarey (American Institute of Physics, Melville, NY, 2009), pp. 297-302.

[42] N.E. Andreev, L. M. Gorbunov, V.I. Kirsanov, K. Nakajima, and A. Ogata, Phys. Plasmas 4, 1145 (1997). 